\title{
Robust grey-box closed-loop stop-and-go control
}

\author{
Jorge Villagra, Brigitte d'Andréa-Novel, Michel Fliess and Hugues Mounier
}

\begin{abstract}
This paper presents a robust stop-and-go control law, especially well adapted to car following scenarios in urban environments. Since many vehicle/road interaction factors (road slope, rolling resistance, aerodynamic forces) are very poorly known and measurements are quite noisy, a robust strategy is proposed within an algebraic framework. On the one hand, noisy signals will be processed in order to obtain accurate derivatives, and thereafter, variable estimates. On the other hand, a grey-box closed-loop control will be implemented to compensate all kind of unmodeled dynamics or parameter uncertainties.
\end{abstract}

\section{INTRODUCTION}

\section{A. Generalities}

Adaptive cruise control (ACC) and stop-and-go control systems have been deeply studied in recent years [17]. Let us recall that while ACC automatically accelerates or decelerates the vehicle to keep a quasi-constant target velocity and headway distance, stop-and-go deals with the vehicle circulating in towns with frequent and sometimes hard stops and accelerations. Both situations present completely different comfort and safety constraints, and therefore, in most of the reported works, ACC and stop-and-go problems are treated separately.

Some approaches ([15], [19]) have tried to reproduce human behavior in order to achieve a 'comfort-based' control. Unfortunately, this kind of strategy may not necessarily lead to safe operation (see e.g. [17]). Besides, external factors such as road characteristics, weather conditions, and traffic load should be taken into account in a robust and safe control system.

Furthermore, an accepted comfort criteria is to guarantee bounded longitudinal accelerations and jerks. Using this idea, many authors (e.g. [1], [2], [6]) have modeled interdistance using different types of time polynomials, whose coefficients are obtained respecting safety acceleration and jerk constraints.

In general, these approaches produce acceptable results in an ACC scenario. However, during a sudden deceleration of the preceding car, the vehicles present a large transitory relative velocity and the actual inter-distance decreases abruptly.

J. Villagra is with the Departamento de Ingenieria de Sistemas y Automatica, Universidad Carlos III, Leganes (Madrid), Spain, jvillagreing.uc $3 m$.es

B. d'Andréa-Novel is with the Centre de Robotique, École des Mines de Paris, 60, bld. Saint Michel, 75272 Paris Cedex, France, brigitte. dandrea-noveldensmp. fr

M. Fliess is with INRIA-ALIEN \& LIX (CNRS, UMR 7161) École polytechnique, 91128 Palaiseau, France, Michel.Fliessepolytechnique.edu

H. Mounier is with the Institut d'Électronique Fondamentale (CNRS, UMR 8622) Université Paris-Sud, 91405 Orsay, France, Hugues.Mounierdief.u-psud.fr
Hence, this dynamical scenario would not be suitably represented by static polynomial models, but by some kind of inter-distance dynamic model.

In [11], the authors proposed a nonlinear reference model taking into account safe and comfort specification in an intuitive way. In addition, the model is combined with a simple feedback loop used to compensate unmodeled dynamics and external disturbances ${ }^{1}$. However, this work makes two assumptions that are never met in real situations:

- the inter-distance and the velocity of the leader vehicle are perfectly measured from suitable sensors;

- the reference acceleration generated by the dynamic inter-distance model is instantaneously applied to the following vehicle.

Our contribution consists in elaborating the engine/brake torque to produce the expected reference acceleration of the follower vehicle, that is, when taking into account measurement noises as well as unmodeled dynamics, such as road inclination, aerodynamic forces or rolling resistance. To achieve this task, a unified approach on estimation and control has been used.

An algebraic framework is proposed to deal with filtering, estimating derivatives, and finally, model free control design.r

It is important to point out that these filters, differentiators and estimators are not of asymptotic nature, and do not require any statistical knowledge of the corrupting noises. This original way of treating conventional problems can be viewed as a change of paradigm in many control and signal processing aspects (cf. [4]).

Finally, in order to minimize the loss of performances due to uncertain road parameters, a model-free control philosophy will be used, which will be adapted by including specific well-known dynamics, in a kind of grey-box model control.

\section{B. Outline of the paper}

The general control scheme will be presented in Section II. In the third Section, the algebraic setting for model-free control will be introduced. Section IV will be devoted to recall the vehicle dynamics and the feedforward control, where a longitudinal acceleration and a consequent torque is generated under ideal circumstances. This section also shows the motivation for the choice of algebraic techniques introduced in Section III. Noise and parameter robustness will be tackled with a grey-box closed-loop control approach in Section IV. Simulation results will show a very good

\footnotetext{
${ }^{1}$ Examples of adaptive car following controllers dealing with this problem can be found in works by [10], [7] or [21].
} 
compromise between performance and robustness. Finally, the conclusion and some future work will be drawn in Section V.

\section{Control SCHEME}

Figure 1 graphically summarizes the whole control scheme. The stop-and-go system uses radar information and $\mathrm{CAN}^{2}$ bus accessible data to generate, via a dynamic model and a reliable follower velocity estimation (see e.g. [18]), the desired acceleration. This model provides a safe and comfortable reference inter-distance between the leader and the following vehicle. A reference longitudinal acceleration is then generated as a feedforward control. Since this model is based on corrupted measures and not always valid assumptions, a feedback term is introduced. This closed-loop will not only behave as a typical PID controller, but it will also estimate linear or non-linear unmodeled dynamics (road slope, wind, rolling resistance) in order to anticipate the controller action. The resulting control will provide an acceleration as close as possible to the desired one.

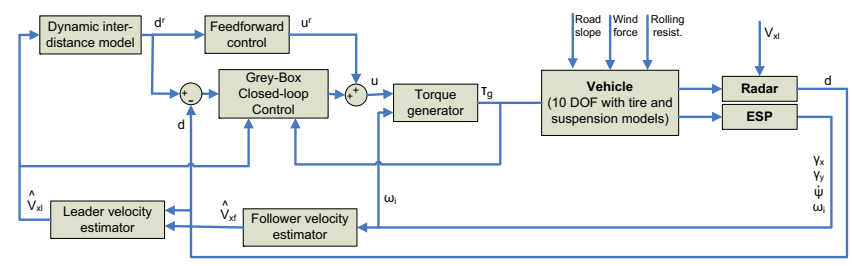

Fig. 1. General Stop-and-go control scheme.

\section{Algebraic SETting FOR MODEL-FREE CONTROL}

\section{A. Numerical differentiation}

Start with a polynomial time function $x_{N}(t)=$ $\sum_{v=0}^{N} x^{(v)}(0) \frac{t^{v}}{v !} \in \mathbb{R}[t], t \geq 0$, of degree $N$. The usual notations of operational calculus (see, e.g., [20]) yield

$$
X_{N}(s)=\sum_{v=0}^{N} \frac{x^{(v)}(0)}{s^{v+1}}
$$

Multiply both sides by positive powers of $\frac{d}{d s}$. The quantities $x^{(v)}(0), v=0,1, \ldots, N$, which are linearly identifiable, satisfy the following triangular system of linear equations:

$$
\frac{d^{\alpha} s^{N+1} X_{N}}{d s^{\alpha}}=\frac{d^{\alpha}}{d s^{\alpha}}\left(\sum_{v=0}^{N} x^{(v)}(0) s^{N-v}\right) \quad 0 \leq \alpha \leq N-1
$$

Multiplying both sides of Eq. (1) by $s^{-\bar{N}}, \bar{N}>N$, permit to get rid of time derivatives, i.e., of $s^{\mu} \frac{d^{l} X_{N}}{d s^{l}}, \mu=1, \ldots, N$, $0 \leq \imath \leq N$.

Consider now an analytic time function, defined by the power series $x(t)=\sum_{v=0}^{\infty} x^{(v)}(0) \frac{t^{v}}{v !}$, which is assumed to be convergent around $t=0$. Approximate $x(t)$ by the truncated

${ }^{2}$ Controller Area Network
Taylor expansion $x_{N}(t)=\sum_{v=0}^{N} x^{(v)}(0) \frac{t^{v}}{v !}$ of order $N$. Good estimates of the derivatives are obtained by the same calculations as above.

Remark 3.1: A most elegant and powerful algorithmic procedure for obtaining a corresponding numerical differentiator is provided in [12]. It will be exploited in the sequel.

\section{B. Model-free control ${ }^{3}$}

Take a finite-dimensional SISO system

$$
E\left(t, y, \dot{y}, \ldots, y^{(l)}, u, \dot{u}, \ldots, u^{(\kappa)}\right)=0
$$

which is linear or not, where $E$ is a sufficiently smooth function of its arguments. Assume that for some integer $n$, $0<n \leq \imath, \frac{\partial E}{\partial y^{(n)}} \not \equiv 0$. The implicit function theorem yields then locally

$$
y^{(n)}=\mathfrak{E}\left(t, y, \dot{y}, \ldots, y^{(n-1)}, y^{(n+1)}, \ldots, y^{(l)}, u, \dot{u}, \ldots, u^{(\kappa)}\right)
$$

This equation becomes by setting $\mathfrak{E}=F+\alpha u$ :

$$
y^{(n)}=F+\alpha u
$$

where

- $\alpha \in \mathbb{R}$ is a non-physical constant parameter, which is chosen by the engineer in such a way that $F$ and $\alpha u$ are of the same magnitude,

- $F$ is determined thanks to the knowledge of $u, \alpha$, and of the estimate of $y^{(n)}$.

Remark 3.2: A system might only be partially unknown as in Sect. IV-E. It is straightforward to adapt the previous method in this case.

In all the known examples until today, $n$ was chosen to be equal to 1 or 2 in Eq. (2). If $n=2$, the desired behavior is obtained via the intelligent PID controller, which is of the form

$$
u=\frac{1}{\alpha}\left(\ddot{y}_{*}-F+K_{P} e+K_{I} \int e d t+K_{D} \frac{d e}{d t}\right)
$$

where

- $y_{*}$ is a reference trajectory

- $e=y-y_{*}$ is the tracking error,

- $K_{P}, K_{I}, K_{D} \in \mathbb{R}$ are suitable gains, the tuning of which is quite straightforward.

\section{HIGH-LEVEL LOOP CONTROL}

The feedforward high level control will be briefly recalled to point out its main features and the fundamental limitations that have been addressed in the present work:

- The closed-loop control is not at all robust to radar noisy measurements.

- When sudden accelerations/decelerations are needed, the corresponding open-loop engine/brake torques may be hard to compute.

After a brief introduction to vehicle longitudinal dynamics, the next subsections will detail how each issue has been addressed, under the algebraic framework presented in Section III.

\footnotetext{
${ }^{3}$ See [3] for more details, for numerous computer simulations, and for
} references on already existing applications. 


\section{A. Vehicle longitudinal dynamics}

A force balance along the vehicle longitudinal axis (cf. [16]) yields

$$
M \ddot{x}=F_{x_{f}}+F_{x_{r}}-F_{a}-R_{x_{f}}-R_{x_{r}}-m g \sin \theta
$$

where $F_{x_{f}}, F_{x_{r}}$ are respectively the front and rear longitudinal tire forces, $R_{x_{f}}$ and $R_{x_{r}}$ the front and rear tire forces due to rolling resistance, $\theta$ the angle of inclination of the road, and $F_{a}$ is the longitudinal aerodynamic drag force.

The rolling resistance forces are often modeled as a timevarying linear function of normal forces on each tire, i.e. $R_{x}=k F_{z}$, with $\mathrm{k}$ the rolling resistance coefficient.

The aerodynamic forces can be written (e.g. [9]) as

$$
F_{a}=\frac{1}{2} \rho C_{d} A_{F}\left(V_{x}+V_{\text {wind }}\right)^{2}
$$

whith $\rho$ being the mass density of air, $C_{d}$ the aerodynamic drag coefficient, $A_{F}$ the frontal area of the vehicle (the projected area of the vehicle in the direction of travel) and $V_{x}, V_{\text {wind }}$ respectively the longitudinal vehicle and wind velocities.

Finally, Pacejka model [14] is used for longitudinal tire/road interaction forces $F_{x}$. They depend on many factors, but essentially on longitudinal slip and normal forces. These normal forces will be computed as realistically as possible within a 10 d.o.f vehicle model (6 d.o.f. of the vehicle center of gravity and one supplementary d.o.f. on each wheel).

\section{B. Feedforward control}

A reference model proposed by [11] will act as a feedforward term into the longitudinal high level control law. The basis of this model will be sketched in the next lines.

The inter-distance reference model describes a virtual vehicle dynamics which is positioned at a distance $d^{r}$ (the reference distance) from the leader vehicle. The reference model dynamics is given by

$$
\ddot{d}^{r}=\ddot{x}_{l}-\ddot{x}_{f}^{r}
$$

where $\ddot{x}_{l}$ is the leader vehicle acceleration and

$$
\ddot{x}_{f}^{r}=u^{r}\left(d^{r}, \dot{d}^{r}\right)
$$

is a nonlinear function of the inter-distance and of its time derivative.

Introducing $\tilde{d} \triangleq d^{0}-d^{r}$ in (5), where $d^{0}$ is the safe nominal inter-distance, the control problem is then to find a suitable control when $\tilde{d} \geqslant 0$ :

$$
u^{r}=u_{2}(\tilde{d}, \dot{\tilde{d}}), \tilde{d} \geqslant 0
$$

such that all the solutions of the dynamics (4) fulfill the following comfort and safety constraints:

- $d^{r} \geqslant d_{c}$, with $d_{c}$ the minimal inter-distance.

- $\left\|\ddot{x}^{r}\right\| \leqslant B_{\max }$, where $B_{\max }$ is the maximum attainable longitudinal acceleration.

- $\left\|\dddot{x}^{r}\right\| \leqslant J_{\max }$, with $J_{\max }$ a bound on the driver desired jerk.
The authors of [11] propose to use a nonlinear damper/spring model $u_{2}=-c|\tilde{d}| \tilde{\tilde{d}}$, which can be introduced in the dynamics equation (4) to give:

$$
\ddot{\tilde{d}}=-c|\tilde{d}| \dot{\tilde{d}}-\ddot{x}_{l} .
$$

The previous equation may be analytically integrated and expressed backwards in terms of $d^{r}$ as follows, assuming that $\dot{x}_{l}(0)=0$ :

$\dot{d}^{r}=\frac{c}{2}\left(d^{0}-d^{r}\right)^{2}+\dot{x}_{l}(t)-\beta, \beta=\dot{x}_{f}^{r}(0)+\frac{c}{2}\left(d^{0}-d^{r}(0)\right)^{2}$.

From (5), the feedforward control law is then obtained applying $^{4}$

$$
\ddot{x}_{f}^{r}=u^{r}=-c\left|d^{0}-d^{r}\right| \dot{d}^{r}
$$

where the inter-distance evolution comes from the numerical integration of (6).

Remark 4.1: In practice, the leader velocity is not measured and we have to construct an estimator, using for example techniques developed in section III.

\section{Closed-loop control}

Some kind of feedback control must be introduced in order to avoid errors induced by measurement noises. A standard PID compensation leads to extremely noisy perturbed results when a derivative term is used, and to instability or important tracking errors when it is not.

In order to avoid this kind of problem, a PD compensator ${ }^{5}$ has been implemented, where inter-distance and its timederivatives are obtained using Sect. III-A. The signal can be locally approximated by a linear polynomial $(N=1)$. Thus, $d(t)=d_{0}+d_{1} t, t \geqslant 0, d_{0}, d_{1} \in \mathbb{R}$. In the first case, an estimator for $d_{0}$ is sought; in the second one, $d_{1}$ will be estimated. If we take for instance $\bar{N}=3$ and $\bar{N}=2$, the estimators can be respectively written as follows :

$$
\begin{aligned}
& \hat{d}=\hat{d}_{0}=\frac{2}{T^{2}} \int_{0}^{T}(2 T-3 \tau) d(\tau) d \tau \\
& \hat{d}=\hat{d}_{1}=\frac{-3 !}{T^{3}} \int_{0}^{T}(T-2 \tau) d(\tau) d \tau
\end{aligned}
$$

Figure 2 shows $^{6}$ the difference between applying two discrete PD controller with a different low-pass filter and an algebraic PD controller.

\footnotetext{
${ }^{4}$ Note that the parameter $c$ is an algebraic function of safe and comfort parameters $d_{c}, V_{\max }, B_{\max }$ and $J_{\max }$ (cf. [11]).

${ }^{5}$ The integral term is not used in order to avoid an unstable behavior of the system (see [11]).

${ }^{6}$ Since the inter-distance reference trajectory depends on the closed-loop behavior, it is difficult to exactly obtain the same testbed for the 3 cases presented in Figure 2.
} 

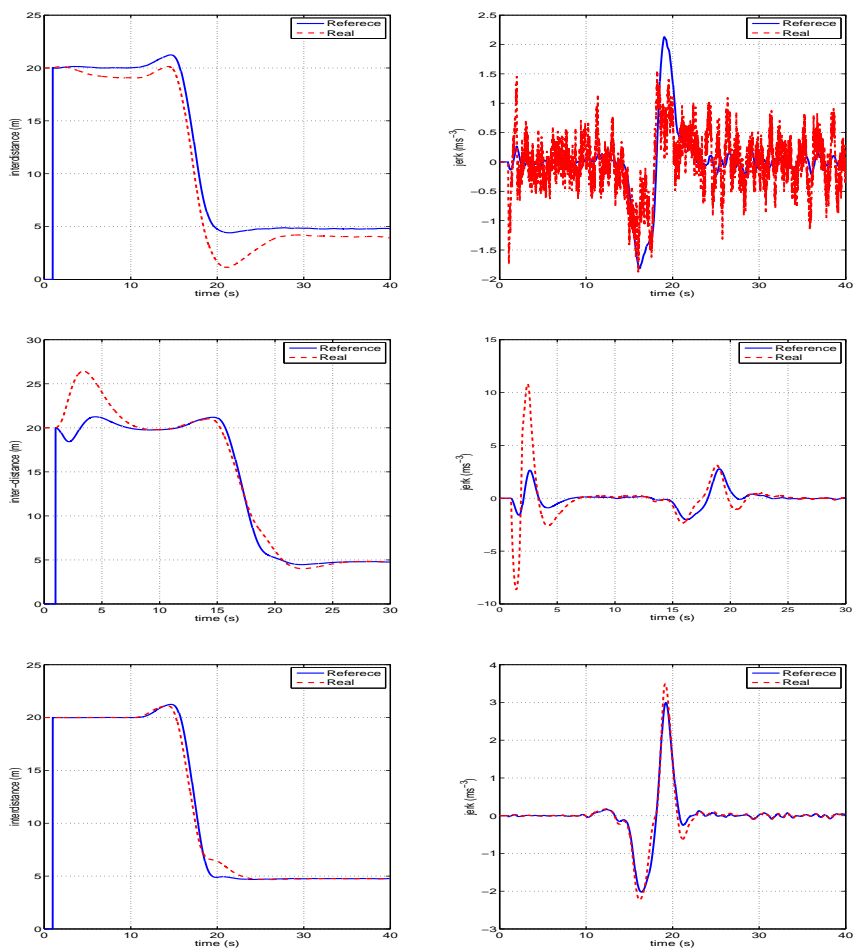

Fig. 2. Inter-distance and jerks evolution with different closed-loop controllers: a discrete PD with low-pass filter of cut-off frequency equal to $100 \mathrm{~Hz}$ (top), another one with cut-off frequency equal to $5 \mathrm{~Hz}$ (middle) and an algebraic PD (bottom). The $P=0.7$ and $D=0.4$ parameters are identical in all cases.

\section{Reference torque generation}

The wheel rotation dynamics can be written as follows

$$
I \dot{\omega}=-r F_{x}+\tau_{e}-\tau_{b}
$$

where $I$ is the rotation inertia moment, $\omega$ the wheel angular velocity, $r$ is the tire radius, $\tau_{e}$ the applied engine torque, and $\tau_{b}$ the brake torque, both of them applied to the wheel center.

A commonly used assumption ([5],[13],[16]) consists in considering rolling without slipping, i.e. $V_{x}=R_{g} r \omega$, where $R_{g}$ is the gear ratio. However, in a stop-and-go context, where fast responses to sudden decelerations are required, this is not an acceptable hypothesis.

If a generalized wheel torque $\tau_{e b}=\tau_{e}-\tau_{b}$ is considered, it is straightforward to see its dependence on tire/road interaction forces. Therefore, a realistic estimation of this generalized torque from equation (9) turns out to be quite hard.

The sum of the 4 wheels rotation dynamics equations and of the vehicle dynamic longitudinal equation $M \gamma_{x}=\sum_{i=1}^{4} F_{x_{i}}$ yields

$$
\tau_{g}=4 \tau_{e b}=I \sum_{i=1}^{4} \dot{\omega}_{i}+r M \gamma_{x}
$$

The main inconvenient for such an estimator is that a good numerical differentiator for $\dot{\omega}_{i}$ is needed. An equivalent algebraic estimator to (8) will then be used to compute $\hat{\hat{\omega}}_{i}$.

Finally, figure 3 compares inter-distances between openloop generated torque under no slipping assumption and open-loop torque with our dynamic estimation approach. A remarkable improvement can be obtained when this new strategy is used in demanding situations. Indeed, results shown in figure 3 are obtained with longitudinal accelerations up to $5.5 \mathrm{~ms}^{-2}$, which are rarely found in an ACC context.

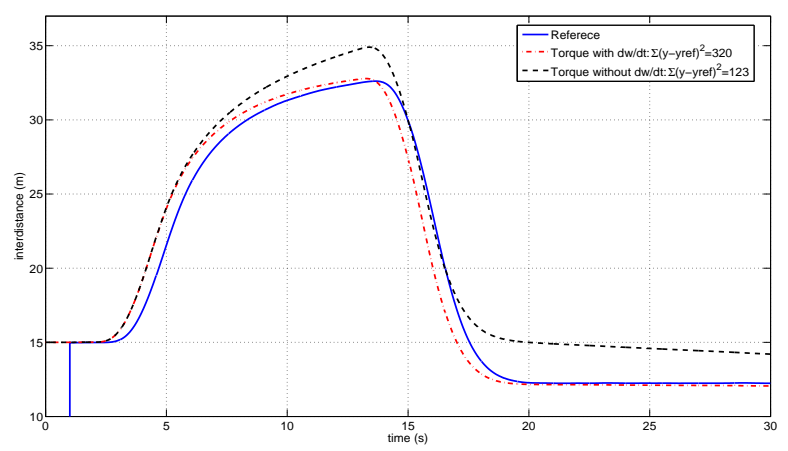

Fig. 3. Comparison between torque generation under no slipping assumption, and with expression (10).

\section{E. Grey-box feedback control}

The procedure described in Sect. III-B can here be applied in a particular way. Since some specific dynamics are very well known, it is worth to integrate them in our predictive scheme. Thus, the design parameter $\alpha$ corresponds here to well-known quantities. Recall the local input-output model introduced in Eq. (2) and compare it with the reordered torque expression (10)

$$
\begin{aligned}
\gamma_{x} & =\frac{1}{M r}\left(\tau_{g}-I \sum_{i=1}^{4} \dot{\omega}_{i}+G(t)\right), \\
G(t) & =r\left(F_{a}-R_{x_{f}}-R_{x_{r}}-M g \sin \theta\right)
\end{aligned}
$$

If rolling without turning is considered $\left(\dot{V}_{x}=\gamma_{x}\right)$, the next equation can then be written:

$$
\dot{V}_{x}=F(t)+\alpha u(t)+\beta(t)
$$

where $F=\frac{G}{M r}, \alpha=\frac{1}{M r}$ and $\beta=-\frac{I \sum_{i=1}^{4} \dot{\omega}_{i}}{M r}$ and $u=\tau_{g}$ is the control variable.

The goal is to obtain an accurate closed-loop estimation of $F$. Following the theoretical ideas described in Sect. III, the procedure consists, first of all, in rewriting (11) in the operational domain, with the assumption $F=F_{0}$ in the estimation time window,

$$
s V_{x}-V_{0}=\frac{F_{0}}{s}+\alpha \tau_{g}(s)+\beta(s)
$$

and then applying the operator $\frac{d}{d s}$ with the aim of eliminating the initial condition $V_{0}$ 


$$
V_{x}+s \frac{d V_{x}}{d s}=-\frac{F_{0}}{s^{2}}+\alpha \frac{d \tau_{g}}{d s}+\frac{d \beta}{d s}
$$

Finally, $s^{-v}$, with $v=2$ is applied in order to eliminate any non causal term

$$
\frac{F_{0}}{s^{4}}=-\frac{1}{s^{2}} V_{x}-\frac{1}{s} \frac{d V_{x}}{d s}+\alpha \frac{1}{s^{2}} \frac{d \tau_{g}}{d s}+\frac{1}{s^{2}} \frac{d \beta}{d s}
$$

which, expressed backwards in the time domain, yields

$$
F_{0}=\frac{3 !}{T^{3}} \int_{0}^{T}\left((-T+2 t) V_{x}(t)-(T-t) t\left(\alpha \tau_{g}(t)+\beta(t)\right)\right) d t
$$

The final closed-loop control is then, applying (3) to our case and considering rolling without turning (i.e. $\dot{V}_{x}^{r}=\ddot{x}_{f}^{r}=u^{r}$ ):

$$
\tau_{g}=M r\left(u^{r}-F_{0}+\frac{I}{M r} \sum_{i=1}^{4} \dot{\omega}_{i}+K_{P} e_{d}+K_{D} \dot{e}_{d}\right), e_{d}=d-d^{r}
$$
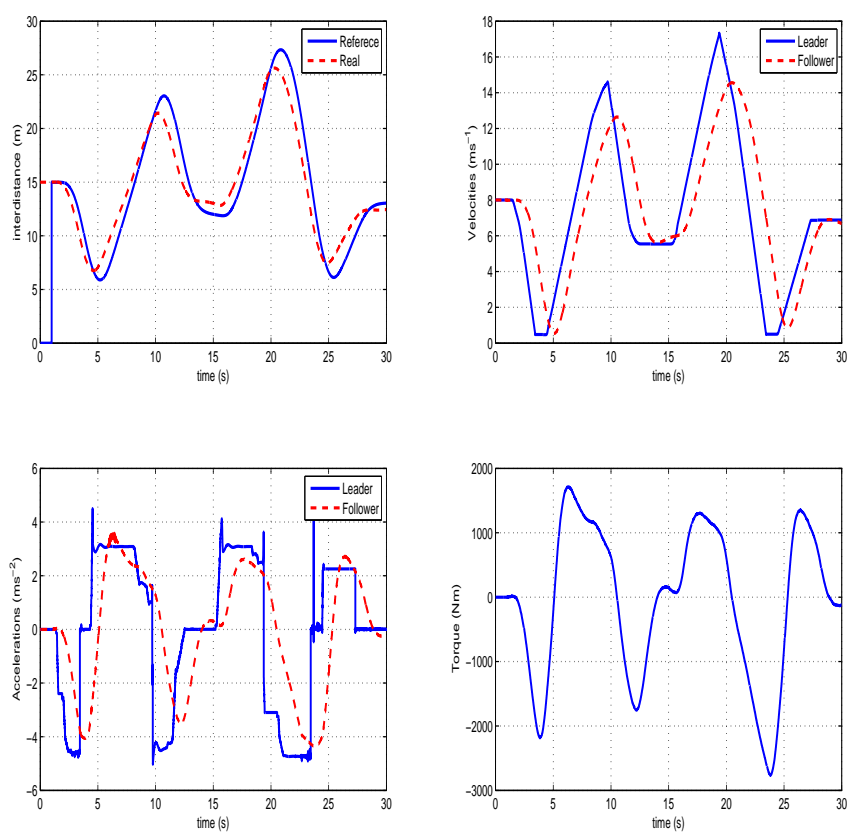

Fig. 4. Inter-distance, velocity, acceleration and generalized torque for highly demanding scenario (up to $5 \mathrm{~ms}^{-1}$ ).

When the complete strategy is tested on a quite demanding scenario $^{7}$, the inter-distance model reference is pretty well tracked (see figure 4), the follower acceleration remains under the comfort constraints, and consequently, the jerk bounds are also guaranteed (cf. [11]). Furthermore, the generalized torque applied to the vehicle seems very robust to noise perturbations.

\footnotetext{
${ }^{7}$ Several heavy accelerations/decelerations are applied to the vehicle on a flat road, where neither rolling resistance nor aerodynamic forces are considered.
}

However, the most important source of uncertainty comes from road conditions. Thus, if rolling resistance, aerodynamic efforts and a sloped road are introduced, the results are slightly different. Figure 5a shows that even if the interdistance trends are already very well respected, a variable bias cannot be annihilated with the "standard" control. The grey-box control strategy has been applied in order to obtain more robust results. The dashed line in figure 5a represents the tracking performance when the estimator $\hat{F}$ of global disturbances is introduced. A considerable improvement (almost $400 \%$ ) is obtained when the global effects of disturbances are estimated via equation (14).
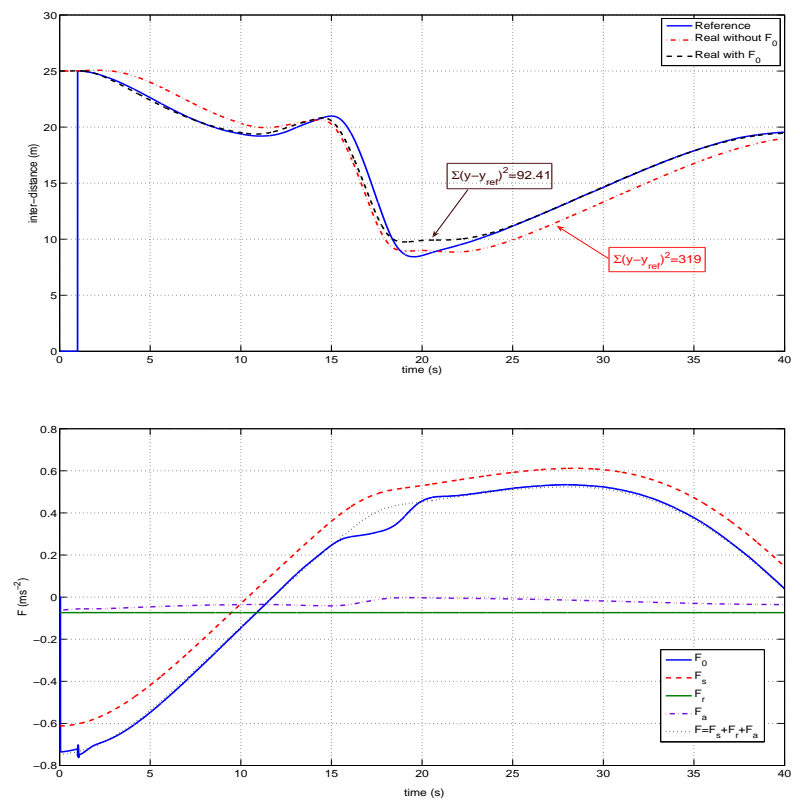

Fig. 5. (a) Inter-distance evolution with and without $F$ estimation. (b) Comparison between real $F$ and its estimate $F_{0}$. Aerodynamic $\left(F_{a}\right)$, road slope $\left(F_{s}\right)$ and rolling resistance $\left(F_{r}\right)$ terms are also depicted.

It can be appreciated from figure 5b that road slope, rolling resistance and aerodynamic forces are pretty well estimated in an overall term $\hat{F}=F_{0}$. Note that aerodynamic forces are not very significant when compared with the road slope. However, big wind gusts can appear at high speeds. In this case, a reliable and fast estimator should applied. Figure 6 shows the behavior of control law proposed in (15) when severe wind gusts longitudinally knock the car. It can be appreciated that our control is much more robust when $F$ is estimated. Moreover, the time window estimation size $T_{e}$ can be used as a tuning parameter for safety or comfort. When tracking performance is more important than comfort (sudden changes in acceleration), a small window will be used. If important jerks are not desired, a bigger window estimation will be more appropriate.

\section{CONCLUDING REMARKS}

A grey-box closed-loop stop-and-go control for vehicles has been presented. Its main feature is its ability to deal with 
usual disturbances (wind, road slope, rolling resistance...) which are not easily measurable. It can be seen that, as expected, our method leads to a closed-loop robust behavior with respect to noises and unmodeled dynamics.

The next step will be to develop a low-level control including the engine and brake dynamics. An algebraic approach is under study to generate the physical control variables: the throttle angle (see already [8]) and brake pressure. Furthermore, the whole algorithm is being adapted to real vehicles and will be soon presented.

\section{REFERENCES}

[1] M. Brackstone and M. McDonald, 'Car-following: A historical review', Transportation Research F, Vol. 2, pp. 181-196, 2000

[2] C. Chien and P. Ioannou, 'Automatic vehicle-following' Proc. Amer. Contr. Conf., pp. 1748-1752, 1992

[3] M. Fliess, C. Join, 'Commande sans modèle et commande à modèle restreint', e-STA, Vol. 5, 2008 (available at http://hal.inria.fr/inria-00288107/en/).

[4] M. Fliess, C. Join, and H. Sira-Ramírez, 'Non-linear estimation is easy', Int. J. Modelling Identification Control, Vol. 3, 2008 (available at http://hal.inria.fr/inria-00158855/en/).

[5] J. K. Hedrick, D. McMahon, V. Narendran, D. Swaroop, 'Longitudinal Vehicle Controller Design for IVHS Systems', Proc. Amer. Control Conf., pp. 3107-3112, Boston, 1991

[6] T. Hiraoka, T. Kunimatsu, O. Nishihara and H. Kumamoto, 'Modeling of driver following behavior based on minimum-jerk theory', Proc. $12^{\text {th }}$ World Congress ITS, paper 3416, San Francisco, 2005.

[7] P. Ioannou and Z. Xu, 'Throttle and Brake Control Systems for Automatic Vehicle Following' IVHS Journal, Vol. 1, pp.345-377, 1994.

[8] C. Join, J. Masse, and M. Fliess, 'Étude préliminaire d'une commande sans modèle pour papillon de moteur', J. europ. syst. automat., Vol. 42, pp. 337-354, 2008.

[9] U. Kiencke and L. Nielsen, Automotive Control Systems, Springer, 2005

[10] M. Liubakka, D. Rhode, J. Winkelman, and P. Kokotovic, 'Adaptive automotive speed control', IEEE Trans. Automat. Control, Vol. 38, pp. 146156, 1993

[11] J. Martinez and C. Canudas-de-Wit, 'A Safe Longitudinal Control for Adaptive Cruise Control and Stop-and-Go Scenarios', IEEE Trans. Control Systems Technology, Vol. 15, pp. 246-258, 2007

[12] M. Mboup, C. Join and M. Fliess, 'A revised look at numerical differentiation with an application to nonlinear feedback control', Proc. $15^{\text {th }}$ Mediterrean Conf. Control Automation, Athens, 2006 (available on http://hal.inria.fr/inria-00142588)

[13] L. Nouvelière and S. Mammar, 'Experimental vehicle longitudinal control using a second order sliding mode technique', Control Eng. Practice, Vol. 15, pp. 943-953, 2007.

[14] H. Pacejka, E. Baker, 'The magic formula tyre model', Proc. $1^{\text {st }}$ Internat. Coll. Tyre Models Vehicle System Analysis, pp. 1-18, 1991.

[15] M. Persson, F. Botling, E. Hesslow and R. Johansson, 'Stop \& Go Controller for Adaptive Cruise Control', Proc. of the IEEE International Conference on Control Applications, pp 1692-1697, Hawaii, 1999

[16] R. Rajamani, Vehicle Dynamics And Control, Springer, 2005

[17] A. Vahidi and A. Eskandarian, 'Research Advances in Intelligent Collision Avoidance and Adaptive Cruise Control', IEEE Trans. Intelligent Transportation Systems, Vol. 4, pp 143-153, 2003.

[18] J. Villagra, B. d'Andréa-Novel, M. Fliess and H. Mounier, 'Estimation of longitudinal and lateral vehicle velocities: an algebraic approach', Proc of 2008American Control Conference, Seattle, 2008 (available at http://hal.inria.fr/inria-00263844/en/)

[19] K. Yi and I. Moon, 'A Driver-Adaptive Stop-and-Go Cruise Control Strategy', Proc. of IEEE Int. Conf. on Networking. Sensing \& Control, pp. 601-601, Taipei, 2004.

[20] K. Yosida, Operational Calculus: A Theory of Hyperfunctions, Springer, New York, 1984 (translated from the Japanese).

[21] K. Youcef-Toumi, Y. Sasage, Y. Ardini, and S. Huang, 'Application of time delay control to an intelligent cruise control system' Proc. American Control Conf., pp. 1743-1747, Chicago, 1992
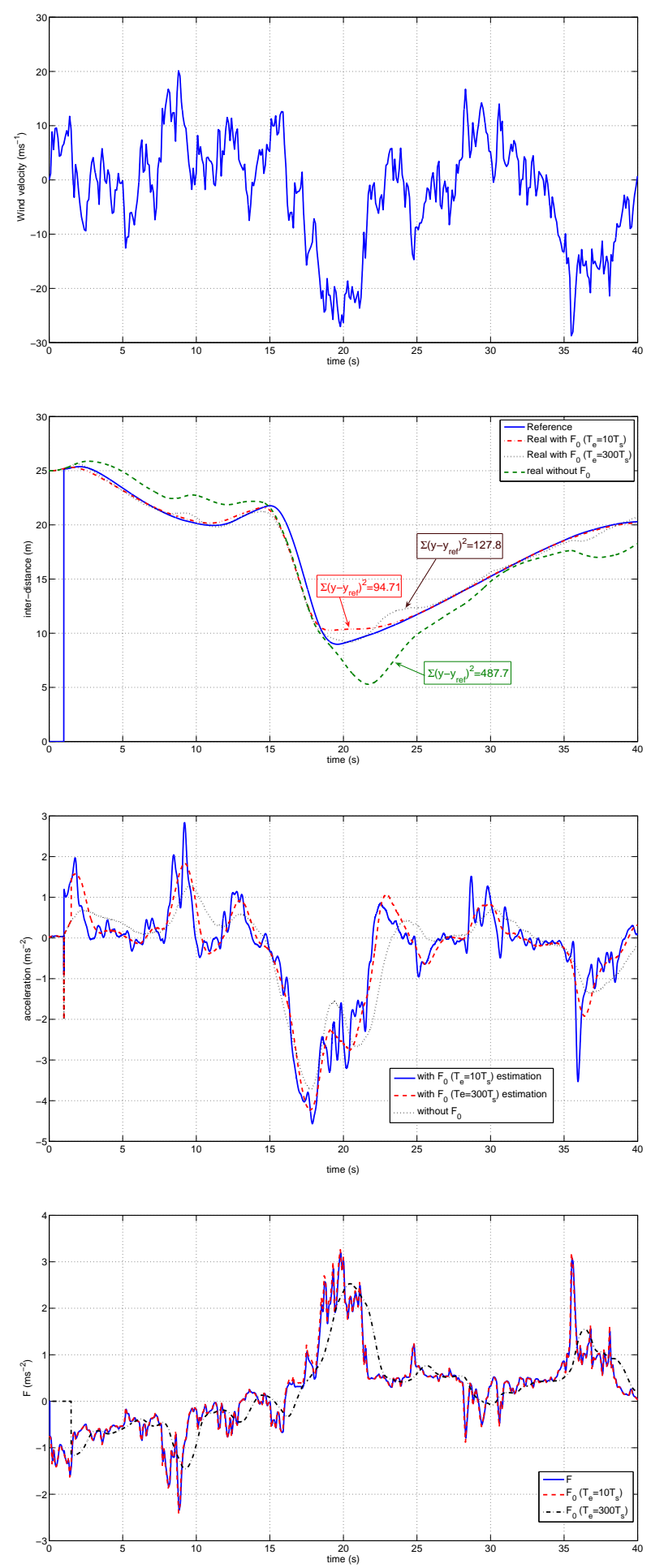

Fig. 6. (a) Wind velocity. (b) Inter-distance evolution with different sliding windows for $F$ estimation. (c) Longitudinal acceleration with different estimation time windows. (d) F estimation 